\title{
EchoGéo
}

22 | 2012

Varia

Vulnérabilité du territoire littoral guyanais aux maladies infectieuses à transmission vectorielle : esquisses de problématiques et perspectives de recherches pluridisciplinaires

\section{Valérie Morel}

\section{OpenEdition}

Journals

Édition électronique

URL : https://journals.openedition.org/echogeo/13275

DOI : $10.4000 /$ echogeo. 13275

ISSN : 1963-1197

\section{Éditeur}

Pôle de recherche pour l'organisation et la diffusion de l'information géographique (CNRS UMR 8586)

Référence électronique

Valérie Morel, « Vulnérabilité du territoire littoral guyanais aux maladies infectieuses à transmission vectorielle : esquisses de problématiques et perspectives de recherches pluridisciplinaires », EchoGéo [En ligne], 22 | 2012, mis en ligne le 30 janvier 2013, consulté le 31 juillet 2021. URL : http:// journals.openedition.org/echogeo/13275; DOI : https://doi.org/10.4000/echogeo.13275

Ce document a été généré automatiquement le 31 juillet 2021.

EchoGéo est mis à disposition selon les termes de la licence Creative Commons Attribution - Pas d'Utilisation Commerciale - Pas de Modification 4.0 International (CC BY-NC-ND) 


\title{
Vulnérabilité du territoire littoral guyanais aux maladies infectieuses à transmission vectorielle : esquisses de problématiques et perspectives de recherches pluridisciplinaires
}

\author{
Valérie Morel
}

\section{Introduction}

1 La chronique des épidémies de maladies infectieuses en Guyane depuis le XVIII ${ }^{e}$ siècle montre combien ce département ultramarin est sensible aux maladies infectieuses à transmission vectorielle (MITV). Si la situation de la Guyane, très proche de l'équateur, par $5^{\circ}$ de latitude Nord définit les éléments du milieu (humidité, chaleur, présence de vecteurs) favorables à l'émergence, ces seuls déterminants ne peuvent rendre compte de la complexité systémique dans laquelle s'inscrit la dynamique des épidémies de MITV.

2 La Guyane fait face à des MITV qui ne constituent pas des priorités pour la métropole. En Guyane, la dengue et le paludisme sont de réels problèmes de santé publique. Le constat d'une distribution spatiale différenciée de ces deux pathologies n'est pas l'unique fait des variations des écosystèmes mais trouve sens dans l'identification de territoire à risques de MITV. Les relations société/territoire contribuent à la construction du système épidémique et dessinent les contours d'une vulnérabilité territoriale différenciée tant dans l'espace qu'à travers le temps-

3 La littérature sur les MITV aborde la vulnérabilité de la société ou d'un groupe face à un risque de MITV par le croisement de données sur l'âge, le genre, les revenus ou encore le niveau social mais plus rarement par les processus territoriaux en action sur l'espace 
qui suggèrent de prendre en compte les usages, la gestion de l'espace, les prises de décisions, la gestion des risques ainsi que les projets de territoire.

En raison des enjeux que représente le littoral pour la Guyane, notamment l'accueil de $80 \%$ de la population, ce territoire s'impose comme terrain d'investigation pour poser les bases d'une réflexion sur les intérêts en termes de recherches et de gestion des MITV. Si le choix actuel des autorités de la sécurité sanitaire est d'optimiser la gestion du risque MITV en ciblant des actions préventives de lutte antivectorielle et d'éducation, étudier le croisement des notions de risque et de territoire permet de faire émerger de nouvelles pistes de recherches et d'actions. La mise en perspective des relations entre MITV et territoire s'inscrit dans la nécessité pour les acteurs de la veille sanitaire de trouver de nouvelles pistes d'action de gestion MITV (Flament et al., 2011) d'autant que les épidémies de dengue de 2006 (Flahaut et al., 2006) et de 2009 ont montré la fragilité du système.

5 Le propos de cet article est de présenter quelques idées de problématique relatives au rapport risque de MITV et territoire sans prétendre en présenter toutes les facettes. Dans cette perspective, une première partie présentera des éléments de contexte en définissant le cadre conceptuel et en rapportant des éléments de la réalité des MITV en Guyane. La seconde partie mettra en exergue l'intérêt de prendre en compte la vulnérabilité territoriale dans la connaissance du système épidémique MITV. Enfin la dernière partie analysera les difficultés de mise en œuvre d'une gestion partagée du risque de MITV et proposera des esquisses de problématiques pour des recherches à venir associant risque de MITV/territoire.

\section{Risque MITV et territoire en Guyane}

6 Malgré près de 65 ans $^{1}$ d'efforts de lutte antivectorielle et de prévention (Cebret, Désiré, 1996), dengue et paludisme persistent en Guyane. En raison du risque sanitaire qu'elles représentent, ces deux maladies font l'objet d'un suivi épidémiologique rigoureux ${ }^{2}$. Avant de restituer des éléments de dynamique de la dengue et du paludisme, il est nécessaire de définir le cadre conceptuel des termes risque et territoire dans le contexte des MITV en Guyane.

\section{Eléments de définition du cadre conceptuel}

7 Il ne parait pas opportun de rapporter ici une synthèse des travaux relatifs aux définitions des notions de risque sanitaire et de territoire, qui pourrait paraître fastidieuse, mais plus de rapporter les spécificités auxquelles renvoient les notions de territoire et de risque dans une démarche de connaissance de la vulnérabilité territoriale au risque de MITV.

\section{Spécificité de la définition du risque MITV}

8 Il faut reconnaître le caractère composite de la définition du risque MITV. Ce caractère composite tient à la participation d'au moins quatre champs d'activités dans la production de données qui participent à la définition opérationnelle du risque de MITV. Il faut distinguer: les sciences biomédicales et les sciences expérimentales qui mobilisent laboratoires et chercheurs dans un domaine particulier comme 
l'épidémiologie, l'entomologie ou encore la modélisation et la spatialisation ; les acteurs de la gestion opérationnelle du risque (ingénieurs et techniciens d'État ou des collectivités territoriales, préfet, élus locaux,...) dont le rapport au domaine précédent est essentiellement d'ordre utilitaire; les médias écrits ou audio-visuels qui jouent un rôle par l'écho qu'ils peuvent donner à une action ou une situation particulière ; et les Sciences Humaines et Sociales (SHS), plus récemment associées et qui montrent que le risque de MITV est aussi un problème social qui recouvre à la fois les attentes de la société, le jeu des acteurs impliqués dans la connaissance et la gestion des MITV et les modalités d'occupation du territoire. Les acteurs appartenant à ces quatre familles d'activités déposent dans le fond de roulement de la connaissance du risque toutes leurs connaissances mais aussi leurs méconnaissances. C'est sous cette dualité connaissance-méconnaissance qu'ils analysent et présentent le risque MITV le plus souvent sous l'angle de l'analyse de l'aléa (les vecteurs) et des dommages (personnes impactées). Une appropriation collective maximale et pluridisciplinaire du risque MITV est nécessaire pour tendre vers une gestion optimale du risque.

\section{Spécificités du territoire dans un questionnement sur les MITV}

Dans l'état actuel des approches en œuvre en Guyane, la problématique risque MITV/ territoire se structure aujourd'hui autour de trois axes majeurs qui découlent du paragraphe qui précède :

- le territoire défini par l'aléa considéré, c'est-à-dire l'espace support du vecteur et de son écologie dont la caractéristique est de se répartir sur des étendues plus ou moins définies et susceptibles de varier dans le temps et l'espace en fonction de dynamiques environnementales et humaines et des connaissances. En Guyane, d'importantes surfaces du département n'ont pas encore été investies par la connaissance entomologique. Il est donc difficile de connaître le niveau d'endémie des MITV. L'absence de données est une réalité qu'il ne faut pas minimiser dans le processus d'analyse ;

- le territoire défini par le périmètre d'action des acteurs gestionnaires du risque, c'est-à-dire un bornage de l'espace par une dimension institutionnelle et opérationnelle. Un grand nombre d'acteurs sur un territoire rend l'action éclatée et polysémique ;

- le territoire défini de façon plus large par les pratiques et représentations des populations, c'est-à-dire-par les diverses formes de relation entre l'espace et la nature avec les individus ou le groupe.

10 Ces trois territoires sont bien évidemment liés les uns aux autres, et interagissent selon une dynamique complexe. La reconnaissance de ces trois territoires par les différents partenaires de la recherche et de l'action se matérialise principalement par la réalisation de cartes qui sont avant tout des cartes de zonage (illustration 1). 
Illustration 1 - Le risque paludique en Guyane, situation en juin 2012

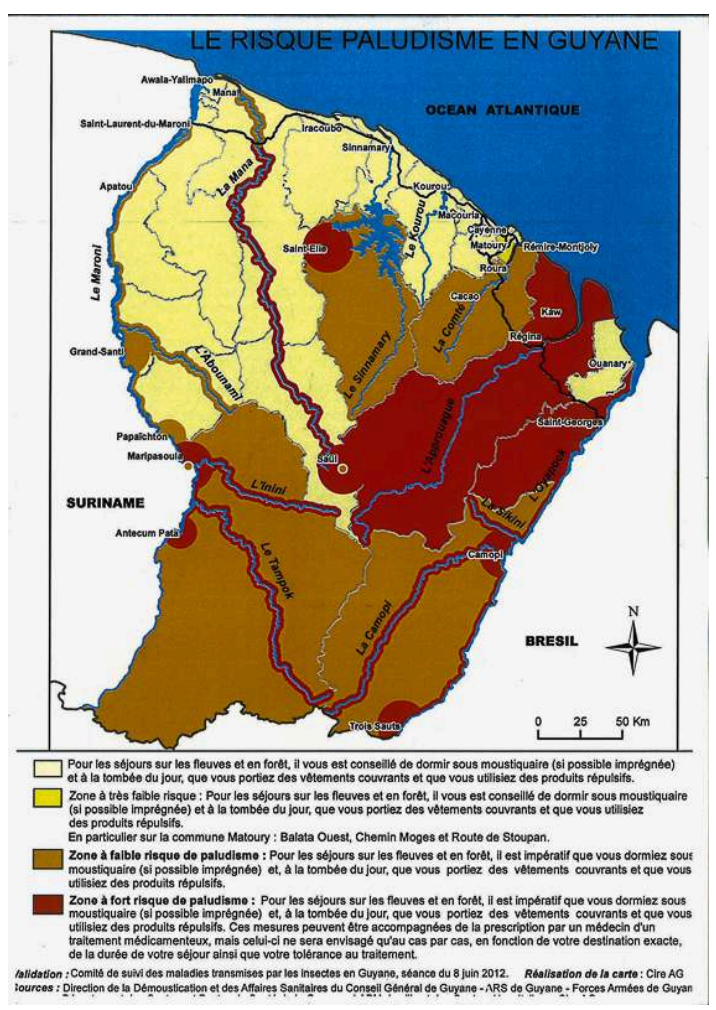

Cette carte est une représentation dans l'espace des zones à risque paludique en Guyane reposant sur la synthèse de trois types de données (nombre de cas, présence de moustiques vecteurs, zone de passage d'orpailleurs) fournies par plusieurs interlocuteurs (Direction de la démoustication et des affaires sanitaires du Conseil général de la Guyane, ARS de Guyane, Forces Armées de Guyane, Département des centres et postes de santé de la Guyane, LABM (Laboratoire d'analyse des biologie médicale) de ville, centres hospitaliers, CIRE Antilles-Guyane). Cette carte est mise et jour et validée tous les trimestres par le comité d'experts des maladies humaines transmises par les insectes. Ce document de spatialisation du risque est un document d'aide à la décision notamment pour la médecine du voyage.

Source : CIRE Antilles Guyane, 2012.

11 La construction des cartes repose sur des caractéristiques entomologiques et épidémiologiques localisées non associées à un fonctionnement territorial individualisé. Les représentations expriment la mise en espace de données dans une vision statique et non dynamique de relations entre MITV et territoire. La confrontation entre données épidémiologiques et analyse géographique du terrain montre que la réalité de la transmission des MITV est modulée en fonction du territoire et des caractéristiques des populations, de leurs modes de consommer l'espace et des modalités de gestion de l'espace et d'appréciation du risque sanitaire. C'est la compréhension de toute cette synergie qui ouvre de nouvelles voies de connaissance et d'actions.

\section{Dengue et paludisme, des maladies endémiques en Guyane}

12 Véhiculée principalement par le moustique Aedes aegypti, la dengue représente la plus fréquente des arboviroses non seulement à l'échelle de la Guyane mais aussi à l'échelle monde. L'Aedes aegypty est présent sur l'ensemble du littoral où il trouve une diversité de gîtes larvaires artificiels favorables à son développement (Fouque, Carinci, 1996). Les 
retours d'expérience des différentes épidémies montrent combien la population du littoral est exposée au risque de dengue. En 2006, une épidémie de dengue était décrite, pour la première fois, à Maripasoula, commune fluviale de l'intérieur du département (Meynard et al., 2008). A partir des années 1940, une campagne d'éradication de l'Aedes aegypti fut mise en œuvre dans tout le département. Cette campagne fut un succès et, en 1950 l'Aedes aegypti était éradiqué en Guyane. En 1963, le suivi entomologique atteste d'une réintroduction de l'Aedes aegypti (Cebert A. et Désiré A., 1996 ; Dussart P., Labeau B., et al. 2006 ; Fouques et Carinci, 1996 ; Reynes, 1996). Dès 1965, les premiers cas de dengue originaires de Guyane sont confirmés conduisant à des épidémies de dengue en 1968-1970, 1972, 1976 et 1982 et par la suite des épidémies de dengue se succèdent tous les 4 à 7 ans associées au cycle épidémique des pays voisins, notamment le Surinam et le Brésil (Failloux et al., 2002).

13 L'éradication du moustique vecteur a été possible entre les années 1930 et 1960 dans une grande partie de l'Amérique centrale, et ce grâce à un effort considérable de lutte antivectorielle conduite par les différents états de la région et coordonnée par la PAHO (Pan American Health Organisation). L'interruption de ces efforts au cours des années 60 a alors permis au moustique de se rétablir. Les pulvérisations à base de DDT utilisées à l'époque pour éradiquer le vecteur ne sont plus envisageables actuellement en raison des effets secondaires tant sur la santé humaine que sur le milieu naturel et du cadre réglementaire européen qui s'applique en Guyane.

Selon le PSAGE dengue (Programme de Surveillance et d'Alerte et de Gestion des Epidémies de dengue) signé en Guyane $2010^{3}$, la dengue circule en Guyane de manière permanente tout au long de l'année et touche particulière les communes littorales urbanisées du département qui se trouvent sur le littoral.

En Guyane, le paludisme est endémique. Au cours des sept dernières années, l'incidence du paludisme a énormément diminué, passant d'un peu plus de 4000 cas en 2005 à près de 1200 cas en 2011 (Ardillon et al., 2012 ; Carme et al., 2009 ; Girod et al., 2011 ; Hommel et al., 1997). Le paludisme est transmis par l'Anophèle darlingi, principal vecteur en Guyane (Girod et al., 2008), implanté à l'intérieur du département et ponctuellement sur le littoral (Pajot et al., 1977). La dynamique du paludisme en Guyane est marquée par des variations dans le temps et l'espace des contextes de risques (Ardillon et al., 2012; Carme et al., 2009; Chocho et al. , 2009 ; CIRE Antilles Guyane, 2006 ; Lepelletier et al., 1989 ; Mouchet et al., 1989). Au début des années 50, le paludisme était fortement implanté sur toute la Guyane. La lutte antipaludique menée entre 1967 et 1978 a été un succès sur le littoral avec l'arrêt de la transmission. Dans l'intérieur du département, peu habité et peu connecté au reste du territoire, l'endémie s'est maintenue. Les bassins fluviaux du Maroni et de l'Oyapock sont des zones d'endémie palustre à transmission permanente (Juminer et al., 1981 ; Lepelletier et al., 1989). Depuis 2005, on observe une évolution ambivalente de la dynamique du paludisme marquée à la fois par la diminution de l'incidence sur l'ensemble du territoire et l'augmentation d'accès palustre sur le littoral. Sur la zone littorale, les cas signalés sont généralement des cas importés des foyers endémiques de l'intérieur, excepté dans certaines zones de transmission autochtone comme certains quartiers de la commune de Matoury ou sur la route de Dégrad Saramaca à Kourou, marquées par des mouvements de populations fréquents avec la zone d'orpaillage de Saint-Elie (Ardillon et al., 2012). 


\section{Inscription des maladies infectieuses sur le territoire littoral}

16 L'émergence des maladies infectieuses sur les espaces littoraux n'est pas un phénomène récent. Les épidémies de maladies infectieuses ont jalonné l'histoire. Par le passé, les questionnements sur les maladies infectieuses abordaient de manière indirecte l'intérêt de l'analyse et de la réflexion des risques sanitaires sur des espaces littoraux. Par exemple, les travaux de l'épidémiologiste Adrien Proust (1883) montrent combien les voies maritimes et les lazarets implantés sur les littoraux sont des éléments clefs dans la diffusion du choléra. Autre exemple, le géographe Maximilien Sorre dans son ouvrage Les fondements de la géographie humaine (1943) met en avant une localisation littorale des foyers de paludisme en France et en Méditerranée. En 1991, l'épidémie de choléra qui a touché les villes portuaires de Chimbote au Pérou et de Guayaquil en Equateur s'est développée sur la frange côtière de ces deux pays. C'est une bactérie probablement originaire d'Asie amenée au Pérou dans l'eau de cale d'un cargo qui par la suite s'est disséminée sur les côtes du Pérou et de l'Equateur par les courants marins (Amat-Roze, 1998; Arbona, Crum, 1996). Plus récemment, les crises du chikungunya à La Réunion (2005-2006) (Taglioni, Deheck, 2009) ou encore l'épidémie de dengue à la Martinique et à la Guadeloupe en 2010 attestent une localisation littorale des bassins à risques de MITV. Ces différents exemples invitent le géographe à rechercher les particularités des littoraux tropicaux face à l'émergence des maladies infectieuses.

Site de plaine côtière baignée par des eaux chaudes et milieu chaud et humide sont des déterminants qui composent le contexte pathogène des espaces littoraux. A cette donne de la nature s'ajoute des facteurs sociétaux liés au pouvoir d'attractivité des côtes et des pôles urbains côtiers dans ces territoires fortement dynamiques. Les villes côtières tropicales comme les villes côtières tempérées sont des espaces de concentration des hommes. Sur les littoraux tropicaux, le système pathogène est très actif en raison de l'explosion démographique qui se matérialise sur l'espace à la fois par une densification des espaces déjà occupés et une expansion urbaine aux dépens des zones de bas-fonds, de la grande mobilité des populations, de la migration des populations, de la variabilité des niveaux de vie, de l'accès à l'eau, de l'installation des lieux de vie dans des environnements neufs ... De plus, ces pôles urbains littoraux tropicaux sont souvent des espaces inscrits dans la dynamique de mondialisation des hommes et des marchandises et des pathogènes par l'intermédiaire d'un port ou d'un aéroport et de trajectoires de migration. La mer et l'air ne sont pas seulement des milieux particuliers, ils sont aussi des routes sur lesquelles ne circulent pas que des hommes, des marchandises et des idées mais aussi des vecteurs de maladies, des microbes, des virus ou des parasites. Tous ces facteurs renvoient à des déterminants qui sont étroitement dépendants des liens qu'entretiennent les individus ou les groupes avec l'espace.

Une entrée géohistorique des maladies infectieuses sur l'espace littoral guyanais montre combien les maladies vectorielles ont marqué la frange côtière guyanaise. Le désastre de l'expédition de Kourou entre 1763 et 1765 à l'origine d'une légende noire de la Guyane en est l'une des premières traces. Une épidémie de fièvre jaune urbaine est souvent mise en avant pour expliquer en partie la mort de 10000 des 12000 embarqués dans cette aventure de colonisation de la Guyane (Ministère de la Marine et des Colonies, 1842). Cet événement peut être interprété comme le signe de la présence, à partir de la fin du XVIII ${ }^{e}$ siècle, de l'Aedes aegypty sur le littoral guyanais, seul vecteur 
de la fièvre jaune reconnu capable de provoquer des épidémies urbaines. L'épidémie de fièvre jaune de 1802 fut la première épidémie cliniquement certifiée de fièvre jaune à Cayenne. En plaçant le compte à rebours de la connaissance des MITV en Guyane au début du XIX ${ }^{\mathrm{e}}$ siècle, nous disposons donc d'une profondeur historique de plus de 2 siècles de retour d'expérience sur les dynamiques spatiales et temporelles des MITV sur le littoral guyanais. Au cours du XIX ${ }^{\mathrm{e}}$ siècle, de nombreux témoignages de fièvre jaune se sont succédés et principalement entre 1850 et 1888. En raison de leur impact sur les populations, la société guyanaise a ressenti le besoin de garder une mémoire de la présence et des impacts des maladies infectieuses sur la population (illustration 2). En 1902, Cayenne fut touchée pour une dernière fois par une épidémie de fièvre jaune urbaine.

Illustration $2 a$ et $2 b$ - Mise en mémoire des dommages causés par l'épidémie de fièvre jaune de 1850-1851, cimetière de Cayenne

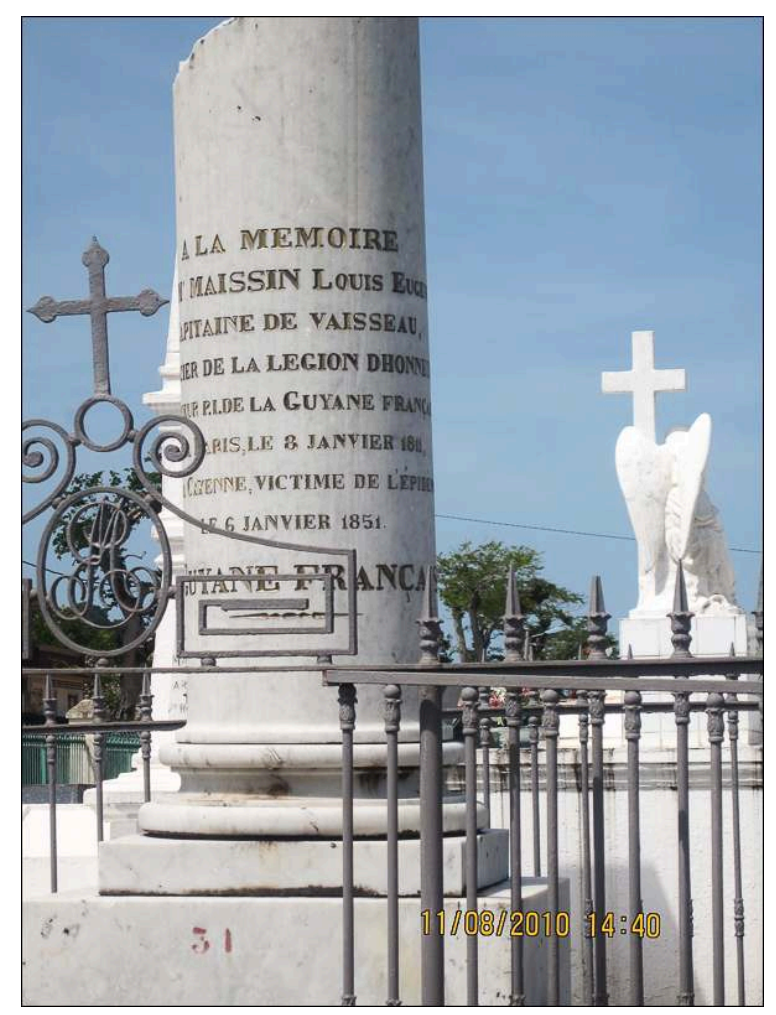




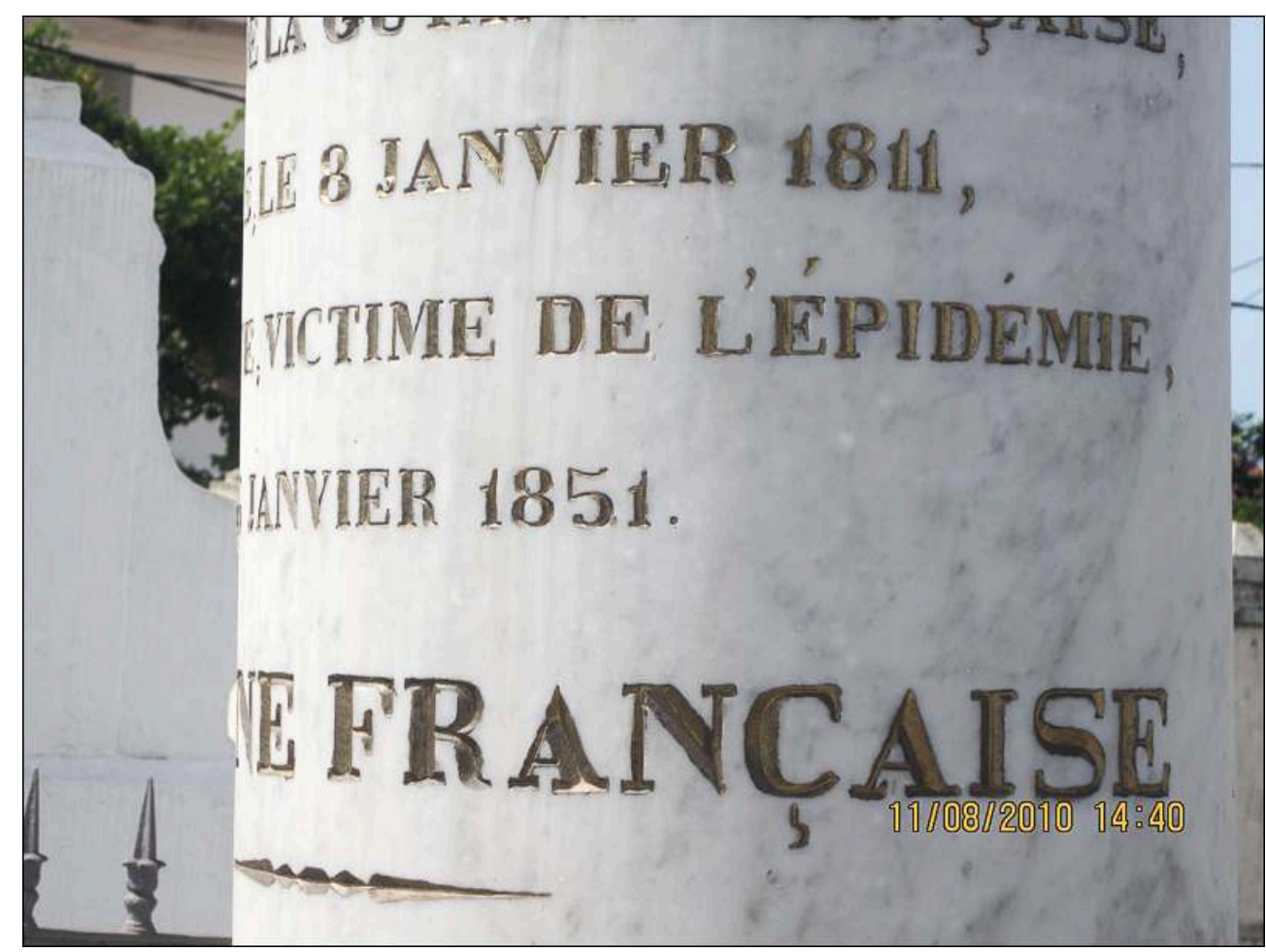

Auteur: V. Morel, 2010.

Si les écrits d'Albert Londres (2002, réédition) «Au bagne» (1923) ou «L'homme qui s'évada " (1928), dont l'intérêt premier est de témoigner sur le fonctionnement de l'institution du bagne, rapportent au lecteur l'hostilité du milieu tropical porteur de maladies qui se manifestent sous la forme de fortes fièvres, une lecture a postériori de ces écrits fait émerger la double réalité d'un littoral vécu comme un milieu malsain et d'une vision éco-centrée des maladies vectorielles.

Les contributions scientifiques produites au cours des dernières années développent progressivement une approche plus anthropocentrée des conditions d'émergence (Ardillon et al., 2012). La dynamique des maladies infectieuses trouve sens non seulement dans la présence de vecteurs mais aussi à travers les comportements humains contextualisés. D'une mise en cause de la nature, c'est dorénavant le fonctionnement $\mathrm{du}$ territoire guyanais par les formes d'urbanisation, certaines pratiques sociales et la mobilité des individus qui définit les conditions de survenue d'une épidémie. D'une certaine manière « habiter le territoire » construit le risque.

21 Tout au long de son histoire, le territoire littoral guyanais s'est vu affecter par des épidémies de MITV marquant les cycles d'occupation de l'espace. Ces épidémies ne sont pas à analyser comme des phénomènes naturels, mais des événements socioenvironnementaux incluant des modalités d'occupation, des activités et des représentations des hommes sur l'espace et la nature.

\section{Vision aléa centrée des MITV en Guyane}

En Guyane, la compréhension de la dynamiques des MITV par les sciences médicales et expérimentales reste très ancrée dans la connaissance de l'aléa, à savoir les agents pathogènes et leurs vecteurs dans leur milieu (Failloux et al., 2002 ; Fouques et Carinci, 
1996 ; Girod et al., 2008 ; Reynes, 1996 ; Meynard et al., 2008 ), et à l'occurrence des cas de MITV confirmés perçus comme des dommages à la population, plus qu'à la compréhension globale du système de production du risque. Les travaux de recherche en sciences biomédicales, en utilisant des enquêtes de type «Connaissance Attitudes et Pratiques » (enquêtes CAP), intègrent de plus en plus des facteurs sociaux caractérisant les populations exposées aux MITV notamment dans la connaissance du paludisme en Guyane (Carme et al., 2002; Stéphani, 2011). L'identification de critères sociaux classiques comme l'âge, le genre, la classe sociale ou le niveau de revenu ne peut définir dans toute sa complexité ce que recouvre la vulnérabilité (Beccera, Peltier, 2009). Les liens entre MITV et construction territoriale ne font pas encore l'objet d'une recherche pluridisciplinaire en Guyane.

Dans ce contexte de connaissances éco-centrées des MITV, il apparait difficile d'échapper à une certaine suprématie de l'aléa (le vecteur) dans l'appréciation du risque de MITV et dans sa gestion. Les producteurs de données spécialisées construisent le risque MITV comme une potentialité de dommage, cas de dengue et de paludisme, dont les origines, les vecteurs, sont extérieurs à l'homme. Une relation causale univoque s'impose: le vecteur touche un groupe social ou un territoire qui subit des dommages. Il apparait alors difficile dans l'esprit des populations et des responsables de la gestion du risque et du territoire de trouver dans l'épidémie d'autres explications que les manifestations d'un phénomène naturel porté à son paroxysme.

\section{Lutter contre les moustiques}

24 La société "agressée » par un agent naturel se défend et met en place des moyens de protection. Les moustiques sont des éléments perturbateurs extérieurs qu'il faut combattre. Pour le risque de MITV, la lutte antivectorielle est la réponse " naturelle » à cette vision et correspond au contrôle des situations d'expositions. Dans cette perspective, la gestion du risque se construit essentiellement sur la connaissance de l'aléa (caractéristiques des vecteurs, localisation, nombre, impact, dynamiques spatiale et temporelle...). C'est en s'appuyant sur ces données que les acteurs de la gestion du risque caractérisent la nature du risque et les solutions devant être apportées notamment en matière de lutte contre les gîtes larvaires portées par la Direction de la Démoustication et des Affaires Sanitaires (DDAES) du Conseil général et des actions de communication à la population (illustration 3). Les modes de gestion des risques de MITV sont déclinés selon un classement académique associant la lutte antivectorielle et la communication. La prévention repose sur la protection individuelle contre les piqûres et le contrôle des populations de moustiques. 
Illustrations ${ }^{\circ} 3$ a et $3 b$ : Affiches de communication et d'éducation sur la dengue dans le quartier informel de Matinhas (Cayenne, mars 2011)
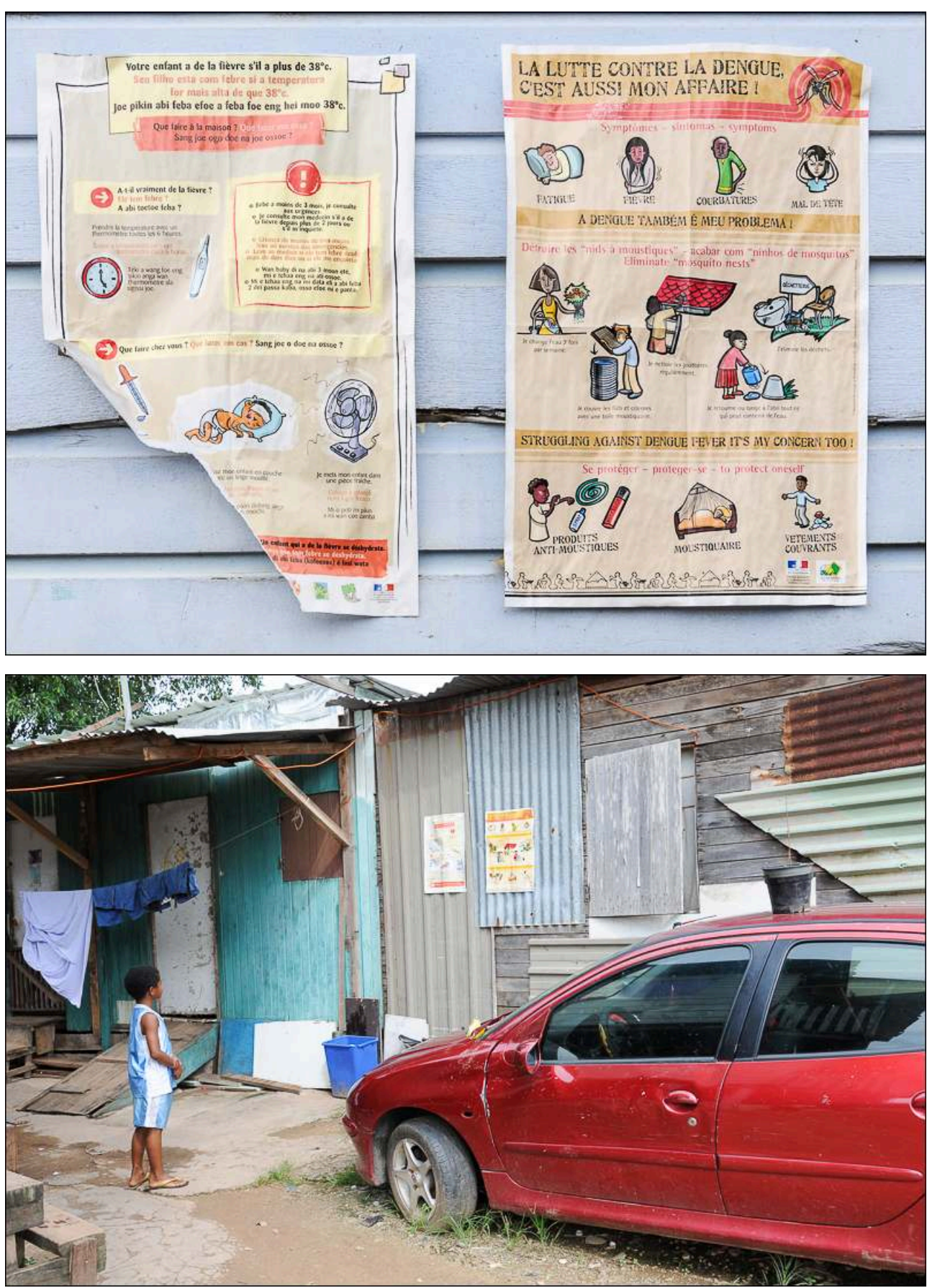

Ce support véhicule un modèle théorique de transmission de la dengue reposant notamment sur des déterminants entomologiques et de protection individuelle qui ne sont pas associés à un territoire en particulier. Alors que la réalité de la transmission est modulée en fonction du territoire et des caractéristiques des populations et de leurs modes d'habiter.

Auteur: V. Morel, 2011

En Guyane, la stratégie et l'organisation actuelles de la lutte contre les vecteurs mises en œuvre par la DDAES sont organisées de manières différentes selon les zones de transmission. Les actions de lutte antivectorielle sont organisées selon un programme fixé annuellement qui dépend essentiellement de la disponibilité des ressources 
humaines et logistiques et ne prend pas en compte d'éventuelles évolutions des MITV sur le territoire. Ponctuellement, des opérations de distribution de moustiquaires imprégnées par des équipes sont mises en œuvre. En juillet 2012, une distribution de moustiquaires s'est déroulée le long des rives françaises de l'Oyapock de Saint-Georges à Trois Sauts avec pour objectif de participer à l'éradication du paludisme à l'échelle du plateau des Guyanes ${ }^{4}$.

Ce dispositif de lutte contre les moustiques est constitutif de la réponse collectivement apportée à la gestion du risque MIT sur le territoire. Une part importante de l'effort d'action est consacrée au traitement des maladies, soit par des actions sur les vecteurs, soit par les soins apportés aux individus. C'est un devoir d'assistance aux populations qui prévaut, la dynamique territoriale n'est pas intégrée à la gestion du risque.

\section{Vulnérabilité territoriale, une vision renouvelée du « contexte pathogène »}

En inscrivant la vulnérabilité dans un cadre territorial, d'une part on renouvelle les perspectives de recherche en associant risque sanitaire et territoire et d'autre part, on montre combien la santé a une place de choix dans la définition d'un projet de territoire.

Le risque de MITV fait appel à la notion de « zone à risque » comme développée dans les travaux du sociologue U. Beck $(1986,2001)$. Aujourd'hui mettre en avant la notion de vulnérabilité territoriale permet de renouveler la notion de "complexe pathogène " élaborée par Maximilien Sorre en 1933 (Sorre, 1933; Le Bras et Malvy, 2004). L'intérêt d'intégrer toute les dimensions de la vulnérabilité pour la connaissance des contextes d'émergence ou de propagation des maladies infectieuses trouve appui dans les récents travaux portés par le Haut Conseil à la santé publique (Leport et Guégan, 2011) où l'intérêt d'un rapprochement entre les sciences médicales et les SHS pour une meilleure connaissance des contextes d'émergence des MITV est mis en avant. "L'intégration des SHS dans la connaissance et la réponse aux situations d'émergence permettra de promouvoir des réponses adaptées aux contextes locaux et régionaux. L'apport des SHS est susceptible d'aider à mieux prendre en compte, tant au plan des populations qu'au plan des ressources socio-économiques et culturelles, certaines "inégalités de vulnérabilité » créatrices d'inégalités entre les territoires » (Leport et Guégan, 2011, p. 20).

Aujourd'hui, reconnaître la part de la vulnérabilité territoriale dans la création d'un contexte de risque sanitaire, c'est analyser la façon dont le territoire, défini comme un construit politique et social (Di Méo, 1998), peut être porteur de compréhension dans le système épidémique. Le territoire n'est pas une zone délimitée administrativement, invariable dans l'espace et le temps. C'est le produit d'une série consciente ou inconsciente d'actions humaines qui influent le système risque sanitaire.

\section{Processus territoriaux en action sur le littoral guyanais}

Avec plus de $80 \%$ de la population vivant sur $5 \%$ du département, le littoral est à la fois «territoire du vide » et « territoire du plein » voire ponctuellement « territoire du trop plein " où la santé est non seulement un enjeu scientifique mais aussi un enjeu politique et social. Il faut aborder le territoire littoral guyanais en tant que territoire 
processus, c'est-à-dire un territoire qui est en pleine transformation, selon des dynamiques démographiques qui modèlent les formes de l'occupation de l'espace et des échelles temporelles et spatiales non seulement variées mais aussi accélérées.

31 Aujourd'hui, la Guyane compte environ 230000 habitants (Insee, 2012) concentrés sur quelques pôles urbains littoraux (illustration 4). La démographie en Guyane n'a cessé d'exploser au cours des 30 dernières années (illustration 5) passant de 67257 personnes en 1980 à 221800 en 2009. Actuellement, le taux d'accroissement de la population est proche de $4 \%$ par an. Cette dynamique démographique est l'expression combinée de l'accroissement naturel $(30 \%)$ et de l'apport migratoire des populations des pays inscrits dans l'environnement régional, soit près de $32 \%$ de la population (Piantoni, 2011).

Illustration 4 - Polarisation de la population en Guyane sur le littoral

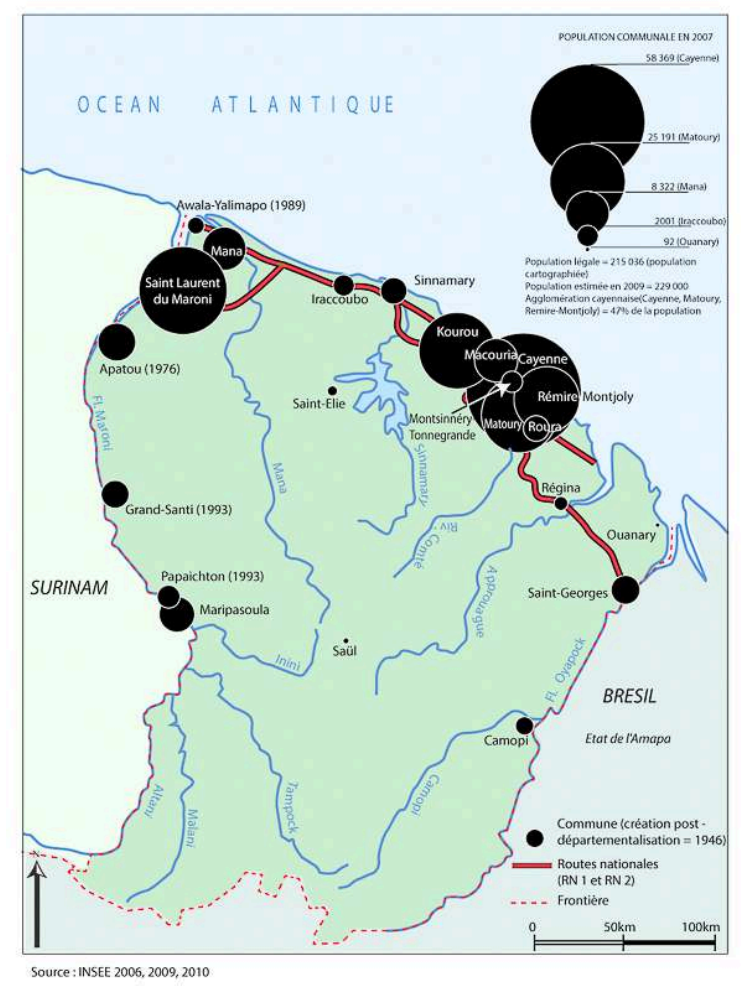

Source : Insee 2006,2009,2010; réalisation : V. Morel. 
Illustration 5 - Croissance de la population de Guyane de 1954 à 2011

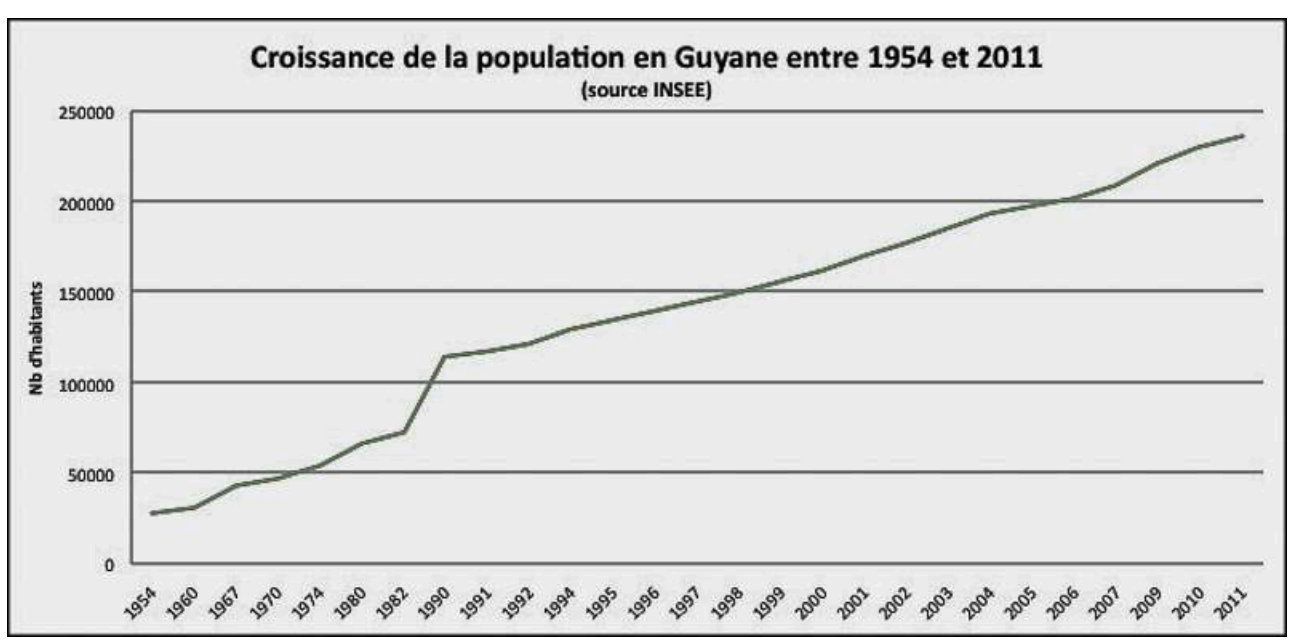

Source : Insee; réalisation: V. Morel.

A la population légale, il faut ajouter une part de population non-régularisée évaluée au quart de la population totale (Piantoni, 2011). Le profil démographique de la Guyane s'apparente à celui des pays en développement dans un contexte économique et politique différent.

Outre la présence du vecteur, l'épidémie de dengue a besoin pour se développer d'un réservoir de population non immune ; lequel se trouve en permanence renouvelé sous l'effet conjugué des deux facteurs socio-démographiques précédemment décrits. De surcroit, il n'existe pas d'immunité croisée entre les différents sérotypes ${ }^{5}$. Ainsi une infection antérieure par un sérotype différent ne protège en rien contre une nouvelle infection, voire même selon certains interlocuteurs est un facteur prédisposant à la survenue de formes graves ou hémorragiques. De même, les personnes venant de métropole pour une période plus ou moins longue constituent également une population sensible. Les différentes formes de renouvellement de la population sont des facteurs d'émergence des contextes à risque.

De plus, la poussée démographique se fait fortement ressentir dans la demande de logements. La pression immobilière touche le littoral où les terres libres sont l'objet de différents conflits d'intérêt (agriculture, protection de la nature, immobilier). L'accès au foncier que ce soit pour l'accès à la ressource ou pour le développement de logements n'est pas aisé. Plus de $90 \%$ des terres font partie du domaine privé de l'Etat, quant au reste des terres, il appartient à quelques propriétaires privés. Dans ce contexte, la forte pression sur le foncier se manifeste par des formes de consommation de l'espace favorables au développement de gîtes larvaires : terrains défrichés laissés à nu et propices à la formation d'étendues d'eau stagnante en saison des pluies, mauvais drainage des terrains dédiés à la construction, occupation de bas-fonds marécageux. Aujourd'hui, on estime que la Guyane accuse un retard de 78000 logements. C'est pour répondre à cette demande que l'État a lancé une action de construction de 3000 logements par an (Ministère de l'Ecologie, de l'Energie, du Développement durable et de la mer, 2010 ; IEDOM, 2010). Les porteurs de projets immobiliers reconnaissent que jamais des réflexions associant maladies vectorielles et organisation et formes du bâti ne sont abordées lors des différentes phases d'élaboration des projets immobiliers. Les lotissements qui sortent de terre sont souvent caractérisés par des densités de bâti 
fortes, où les maisons sont souvent mitoyennes, où parfois un bassin de rétention des eaux pluviales à ciel ouvert peut être implanté en pleine zone bâtie et encore en lisière de zones marécageuses. Ces formes et modalités d'urbanisation participent à la dynamique des épidémies de MITV. En parallèle de cette politique du logement qui ne peut satisfaire tous les besoins, des familles sans terre s'installent sur des espaces marginaux. De nouveaux quartiers informels participant à l'extension de l'urbanisation apparaissent de manière spontanée. L'urbanisation croissante, l'exposition à des zones d'aléas marquées par des densités vectorielles importantes et la concentration de plus en plus forte des populations sont des facteurs d'émergence de la dengue que majorent la promiscuité et la multiplication des contacts humains.

Quant au paludisme, le nombre de cas sur le littoral augmente. Les cartes et les données publiées dans les Bulletins Mensuels de la Veille Epidémiologique laissent apparaitre l'éventualité de foyers littoraux de paludisme (Ardillon et al., 2012). Des éléments d'explication sont à chercher d'une part, dans les usages de l'environnement familier et d'autre part, dans la mobilité des populations d'orpailleurs arrivant des pays limitrophes impaludés (Brésil et Surinam) et exploitant des placers illégaux en forêt. Des flux de main-d'œuvre clandestine installée à l'intérieur de la Guyane empruntent les fleuves pour rejoindre des noyaux de solidarité installés sur le littoral et ainsi participent à la diffusion du paludisme.

Depuis 2003, la réalisation de la route reliant Saint-Georges à Régina facilite les déplacements de population tant légale qu'illégale non seulement dans l'est guyanais mais aussi entre l'intérieur et le littoral du département. Circulation des populations, conditions de vie précaires sur les placers (Bourdier, 2004) et difficultés d'accès aux soins et à la prévention favorisent la réactivation d'anciens foyers paludiques comme à Régina ou à Saint-Elie, ou l'éclosion de nouveaux. Dans la zone côtière et les environs de Cayenne, 300 à 400 cas de paludisme ont été déclarés annuellement en 1999 et 2002, mais seulement $10 \%$ à $20 \%$ d'entre eux doivent être considérés comme des cas «autochtones ». La transmission du paludisme s'effectue de manière sporadique dans les secteurs et quartiers très localisés où il existe des flux de populations venant des zones de transmission ou des pays voisins.

37 Aujourd'hui, l'intérieur de la Guyane est face à un processus de consommation de l'espace et d'anthropisation de la forêt qui provoque non seulement d'importantes modifications de l'écosystème consécutives au développement de l'orpaillage illégal mais qui participe aussi à la mise en connexion plus fréquente de l'intérieur du département avec le littoral.

\section{Gérer ensemble les MITV}

Au regard des enjeux des maladies infectieuses, la CIRE $^{6}$ Antilles Guyane affiche dans un récent travail la nécessité de repenser le dispositif de surveillance épidémiologique des maladies infectieuses et la nécessité d'élaborer un outil permettant de rassembler et d'harmoniser les données produites par l'ensemble des acteurs et ainsi de coordonner l'ensemble des partenaires au sein d'un réel réseau de suivi des différentes pathologies (Flamand et al. 2011). L'intégration d'un volet vulnérabilité territoriale croissant risque de MITV/territoire dans une action de réorganisation de la veille construit autour de l'outil SISMIP (Système d'Information pour la Surveillance des Maladies Infectieuses 
Prioritaires) apporterait un nouveau volet à la compréhension des systèmes épidémiques.

\section{Facteurs de vulnérabilité et modes d'appropriation des MITV par les acteurs du territoire}

39 A la lumière des travaux de J.-Cl. Thouret et R. D'Ercole (1996) sur l'identification des « facteurs de vulnérabilité » propre aux modes de gestion des risques naturels dans des contextes territoriaux connus, nous avons entrepris d'analyser les modalités d'appropriation collective et pluridisciplinaire des MITV par les acteurs du territoire. Ainsi la construction territoriale spontanée et planifiée met en œuvre des logiques d'actions et de relations qui peuvent se transformer en facteurs actifs de vulnérabilité.

\section{Acteurs variés, une difficile convergence des logiques d'action}

\section{complémentaires, prenant en compte, d'une part, la dimension matérielle du territoire,} objet physique à analyser dans son fonctionnement et ses transformations et d'autre part, sa dimension immatérielle, objet vécu et perçu par le biais des représentations. Parallèlement aux données empiriques mobilisées par la collecte de documents de planification de l'espace, d'action en termes de connaissances et de surveillance des MITV ou encore le traitement de données statistiques, des données plus qualitatives ont été recueillies par questionnaires auprès de la population et des entretiens semidirigés menés auprès de la majorité des acteurs impliqués ${ }^{7}$ entre 2010 et 2011. Dans certains contextes localisés et notamment dans les quartiers informels, le questionnaire s'est révélé inadapté pour approcher toute la complexité de la construction d'un espace à risque sanitaire autour de la problématique maladies infectieuses à transmission vectorielle. Une nécessaire immersion dans ces quartiers s'imposait pour gagner la confiance des habitants et recueillir des données qualitatives indispensables à la compréhension des relations entre une population composite et les maladies infectieuses. L'emploi du questionnaire étant trop lisse et trop normalisé pour des espaces où les normes en action ne sont pas celles d'une vision régalienne du fonctionnement d'un territoire et où la marginalisation territoriale, politique, sociale, économique et sanitaire est une réalité quotidienne.

\section{Appropriation collective des MITV}

Le traitement des entretiens et des questionnaires apporte un éclairage sur l'appropriation collective du risque de MITV, qui selon notre hypothèse, joue un rôle non négligeable dans la reconnaissance de la complexité du système risque de MITV. Les informations collectées ont été analysées selon trois idées forces à savoir: la définition du territoire à risque de MITV, les modalités d'appropriation du territoire par chaque acteur, et la mise en réseau des acteurs. 
Il s'agit donc d'identifier et d'articuler les logiques des différents groupes acteurs dans la prise en compte des relations risques de MITV et territoire (illustration 6).

L'analyse du terme territoire montre qu'il s'impose comme un concept polysémique, multiscalaire, et plurifonctionnel. Le territoire est à la fois, un milieu, un écosystème, un périmètre intervention, un établissement, une entité administrative ou encore un espace de vie. Bien qu'un caractère multidimensionnel s'impose, on constate que chaque acteur conserve sa propre définition du territoire. Dans ce contexte, la mise en réseau de la connaissance et de l'action pour dépasser la vision univoque de chaque acteur et afin de rendre compte de la complexité territoriale du système risque paraît difficile.

De plus, les acteurs investissent et s'approprient le territoire MITV par la mise en œuvre de compétences bien déterminées (connaissance, action politique, coordination, pression, action à la personne, équipement...) qui ne sont pas toujours abordées sous l'angle de l'interdépendance des actions. Enfin, on constate que la mise en réseau des acteurs est peu opérationnelle car chaque groupe d'acteurs agit en fonction d'une logique qui constitue son cœur de métier. Quatre types de logiques ont pu être identifiées: logique sécuritaire préalable, logique sécuritaire d'intervention, logique sécuritaire de prévention et logique d'aménagement. On constate que ces logiques sont communes voire partagées entre deux ou trois acteurs, rarement plus. Dans ce contexte, un fonctionnement en réseau des différents acteurs n'est pas encore une réalité opérationnelle.

Illustration 6 - Territoire et logique d'action des acteurs sur la thématique du risque de MITV en Guyane

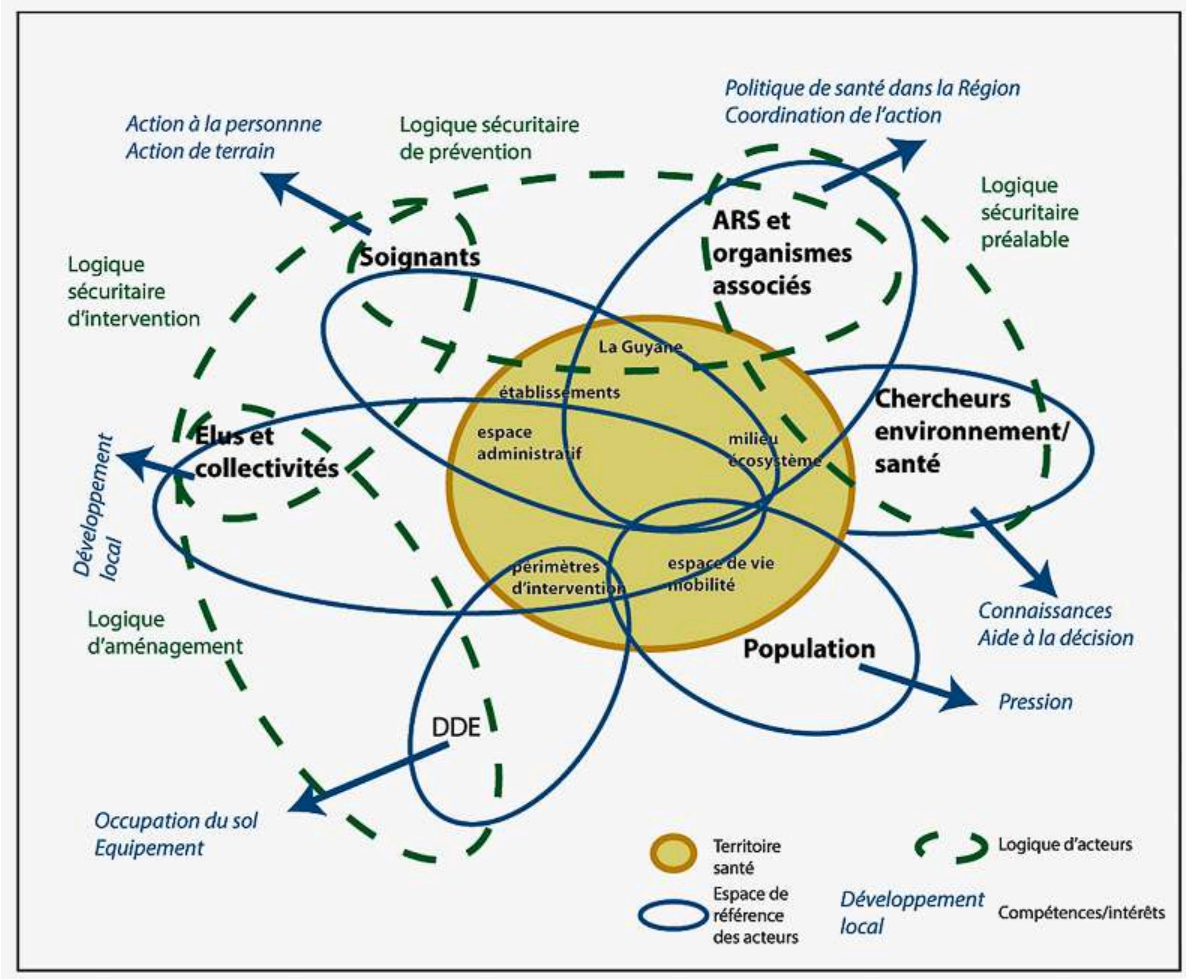

Ce schéma a été élaboré à partir de données recueillies en Guyane avant la réorganisation des services de l'État, effective depuis janvier 2011.

Réalisation : V. Morel. 
Les divers acteurs qui participent au binôme territoire-MITV ont tous une perception différenciée du lien territoire-santé et des imbrications possibles. En ce sens, il est possible d'avancer que le couple territoire-MITV possède un "spectre sémantique " dans lequel chaque acteur possède sa «longueur d'onde». Si aucun acteur n'ignore totalement les centres d'intérêt des autres, chacun raisonne en fonction de son ordre de priorité. Chaque acteur a une vision fragmentaire du système risque MITV ce qui, d'une part, rend la Guyane vulnérable aux MITV et, d'autre part rend difficile la mise en œuvre d'une gestion partagée et contextualisée des MITV en Guyane.

\section{Pistes de recherche pluridisciplinaire}

Du point de vue opérationnel, bien des défis sont à dépasser pour que les programmes de recherche et la gestion du risque de MITV soient réellement pluridisciplinaires et contribuent effectivement à réduire la vulnérabilité territoriale aux MITV.

Le premier effort est de dépasser les frontières disciplinaires et d'aller non seulement vers des recherches pluridisciplinaires mais aussi des comités d'experts pluridisciplinaires. Introduire des experts en SHS dans le comité d'experts des maladies humaines transmisses par les insectes serait un premier pas vers la pluridisciplinarité. Ce premier partage des connaissances, des données, des méthodologies et des incertitudes scientifiques permettrait réellement de travailler sur le risque dans son acception la plus globale. Aujourd'hui, les différents acteurs intéressés par la gestion des MITV développent un emploi abusif du terme risque dans la formulation de leur travaux ou leur action de terrain, bien souvent recherche et action interrogent et agissent uniquement sur l'aléa et la mesure des dommages.

Un autre défi sera de créer des passerelles entre la recherche et les opérateurs du territoire pour faire passer l'idée que la transmission des MITV n'est pas à analyser uniquement comme un phénomène naturel mais aussi comme une succession d'événements socio-environnementaux qui évoluent dans le temps et l'espace. Pour ce faire, il reste à démontrer que les SHS sont tout autant que les sciences biomédicales et expérimentales des disciplines de l'opérationnalité et de l'action publique. De plus, il faudrait introduire dans l'élaboration des projets de territoire une réelle réflexion sur les liens entre aménagement du territoire et transmission des MITV. Ces réflexions partagées pourraient être force de proposition dans les documents de planification de l'espace.

50 Enfin, à l'heure où les pouvoirs publics entendent développer la participation citoyenne, il faut intégrer réellement l'ensemble des populations dans leur diversité, leurs attentes et leurs connaissances pour tendre vers une gestion intégrée des MITV. Intégrer la population est le meilleur levier pour rendre une politique de gestion d'un risque opérationnelle et mettre en œuvre les stratégies de mitigation ${ }^{8}$ les plus adaptées au contexte local.

\section{Conclusion}

51 L'approche par la vulnérabilité territoriale des MITV se trouve à la confluence de deux schémas de pensée et d'action: l'ancien, celui du curatif et de l'approche 
principalement médicale et naturaliste et le nouveau, celui de la société du risque où anticipation et pluridisciplinarité s'associent.

Cette reconnaissance est lourde de conséquences et implique toute une série de changements. Parmi eux, l'évolution des schémas cognitifs et normatifs dans les milieux décisionnels, l'assouplissement des routines institutionnelles et l'ouverture d'espaces de discussion, la requalification des connaissances, la réadaptation des grilles d'analyse. Une approche du système épidémique par la vulnérabilité territoriale est d'autant plus légitime que la rapidité de transformation du territoire littoral guyanais favorise la construction de territoires à risque de MITV variés et imbriqués.

Les porteurs de la sécurité sanitaire sont interpelés par la dynamique actuelle des MITV qui représentent des enjeux sociétaux et politiques dans des territoires de plus en plus connectés (Bley, 2010) où le littoral constitue une réelle porte d'entrée pour les hommes et les vecteurs. A cet égard, soulignons que les risques de MITV ne concernent pas uniquement les $\mathrm{DFA}^{9}$. En métropole, le risque épidémique de dengue s'ancre en France métropolitaine. Depuis 2004, un moustique du genre Aedes, Aedes albopictus, également vecteur de la dengue, s'est progressivement installé en France métropolitaine. En 2011, ce moustique était implanté dans huit départements méditerranéens littoraux : Alpes-de-Haute-Provence, Alpes-Maritimes, Bouches-duRhône, Corse-du-Sud, Haute-Corse, Var, Gard et l'Hérault (INVS, 2011). Du fait de la présence de ce moustique dans ces départements, le risque de déclenchement d'une épidémie à partir de cas importés est devenu réel en métropole. Le risque de transmission des MITV et notamment de la dengue en métropole doit donc être replacée dans un contexte épidémiologique imbriqué non seulement entre les DFA et la métropole mais aussi à l'échelle d'un contexte épidémiologique mondial. A cet égard, la connaissance, dans toute sa complexité, de la vulnérabilité territoriale de la Guyane aux MITV présente un intérêt majeur non seulement à l'échelle du département mais aussi outre atlantique en raison de l'importance et de la fréquence des échanges de biens et de personnes entre la Guyane et la métropole.

Cette publication s'inscrit dans les travaux du Laboratoire d'excellence CEBA (ANR-10- LABX-25).

\section{BIBLIOGRAPHIE}

Abrona S. \& Crum S., 1996. Medical geography and cholera in Perú. http://www.colorado.edu/ geography/gcraft/warmup/cholera/cholera_f.html

Ardillon V., Eltges F., Chocho A., Chantilly S., Carvalho L., Flamand Cl., Carme B., 2012. Evolution de la situation épidémiologique du paludisme en Guyane de 2005 à 2011, BVS, 1-2, p 5-11.

Amat-Roze J.-R., 1998. Risques sanitaires et territoires à risque : perception individuelle et perception collective, du groupe à l'État. In Guillaud D. (ed.), Seysset M. (ed.), Walter A. (ed.), Le voyage inachevé ... à Joëlle Bonnemaison. Paris, ORSTOM, Prodig, p. 543-550.

Beccera S., Peltier A., (dir.), 2009. Risques et environnement : recherches interdisciplinaires sur la vulnérabilité des sociétés. L'Harmattan, 575 p. 
Beck U., 2001, La société du risque. Sur la voie d'une autre modernité. Paris, Flammarion, coll. Campus, $521 \mathrm{p}$.

Beck E, Glatron S., 2009. Vulnérabilité socio-spatiale aux risques majeurs : l'approche du géographe. In Beccera S., Peltier A., Risques et environnement : recherches interdisciplinaires sur la vulnérabilité des sociétés, Paris, L'Harmattan, p. 331-346.

Bley D., 2010. Les maladies à transmission vectorielle, pourquoi s'y intéresser ?, Natures Sciences Sociétés, 18, p 101-102.

Bogardi J.J., 2004. Hazards, risks and vulnerabilities in a changing environment: the unexpected onslaught on human security. Global Environmental Change, 14, p. 361-365.

Bourdier F., 2004. Migration et sida en Amazonie française et brésilienne. Matoury, Ibis Rouge Editions, coll. Espace Outre-Mer, 250 p.

Carme B., Pignaux R., Lecat J., 2002. Connaissance, attitudes et pratiques vis-à-vis du paludisme des populations dans les régions d'endémie en Guyane. Troisième conférences de consensus sur le paludisme en Guyane, Cayenne, octobre, p. 21-22.

Carme B., 2005, Epidémiologie et diagnostique du paludisme en Guyane. Revue Francophone des Laboratoires, 374, p. 21-25.

Carme B., Ardillon V., Girod R., Grenier C., Joubert M., Djossou F., Ravachol F., 2009. Situation épidémiologique du paludisme en Guyane. Médecine Tropicale, 69, p. 19-25.

Cebret A., Désiré R., 1996. La lutte anti-stégomyienne en Guyane française. Bulletin de la Société des Pathologies Exotiques, 89, 2, p. 148-153.

Chocho A., Bellony S., Azor P. Chantilly S., 2009. Lieux présumés de contamination palustre répertoriés sur le littoral de la Guyane. $B V S, n^{\circ} 1,15 \mathrm{p}$.

CIRE Antilles Guyane, 2007. Situation épidémiologique du paludisme en Guyane (novembre 2006). BASAG, $1,13 \mathrm{p}$.

CIRE Antilles Guyane, 2011. Surveillance du paludisme. Le point épidémio, 4, 3 p.

CIRE Antilles Guyane, 2011. Surveillance de la dengue. BVS, 5, 3 p.

CIRE Antilles Guyane, 2012. Spécial paludisme dans les Antilles et en Guyane. BVS, 1-2, 26 p.

Cohen M.L., 2000. Changing patterns of infectious disease. Nature, 406, p. 762-767.

Daszak P., Cunningham A.A. \& Hyatt AD., 2000. Emerging infectious disease of wildlife: threats to biodiversity and human health. Science, 287, p. 443-449.

Daszak P., Cunningham A.A. \& Hyatt AD., 2001. Anthropogenic environmental change and emergence of infectious diseases in wildlife. Acta Tropica, 78, p. 103-116.

D’Ercole R., 1991. Vulnérabilité des populations face au risque volcanique. Le cas de la région du volcan Cotopaxi (Equateur). Thèse de doctorat, Université J. Fourier, Grenoble, 460 p.

D’Ercole, R. 1994. La vulnérabilité des sociétés et des espaces urbanisés : concepts, typologie, modes d'analyses. Revue de Géographie Alpine, vol. 82, n4, p. 87-96.

D’Ercole R., Thouret J.-C., Dolfus O., et Asté J.P., 1994. Les vulnérabilités des sociétés dans des espaces urbanisés : concepts; typologie, modèles d'analyse. Revue de Géographie Alpine, 82, 4, pp. 87-96.

Di Méo G., 1998. Géographie sociale et territoires. Paris, Nathan 317 p. 
Eve M.-E., 2006. Risques sanitaires et gestion des ressources. Ceras, Projet, n²93, juillet 2006. URL : http://www.ceras-projet.com/index.php?id=983

Fabiani J.L., Theys J., 1987. La société vulnérable, évaluer et maîtriser les risques. Paris, Presse de l'Ecole Normale Supérieure, $674 \mathrm{p}$.

Fabiani J.-L., 2010. Repères : risques et environnement : recherches interdisciplinaires sur la vulnérabilité des sociétés. Natures Sciences Sociétés, 18, p. 473-496.

Failloux A.-B, Fouque F., Vazeille M., Rodhain F., 2002. Isoenzyme différentiation of Aedes aegypti populations in French Guiana. Medical and Veterinary Entomology, 16, p. 456-460.

Flahaut A., Lotteau A., Favier F., Trouvin J.H., Kougard J.M., 2006, Mission de recherche sur la dengue en Guyane française. Rapport de mission $n^{\circ} 5$ (02-06 mai 2006). Ministère de la Santé et des Solidarités, $14 \mathrm{p}$.

Flahaut A., Zyberman P., 2008. Des épidémies et des hommes. Paris, Editions de la Matinière, 238 p.

Flamand Cl., Galarraga D., Eve R., Ardillon V., Carvalho L., Marques F., Quénel F., 2011. SISMIP Guyane, un outil dédié à la surveillance épidémiologique des maladies infectieuses prioritaire en Guyane. BVS, 6, p. 2-7.

Fouque F., Carinci R., 1996. Aedes Aegypti en Guyane française. Quelques aspects de l'histoire, de l'écologie et de la transmission verticale des virus de la dengue. Bulletin de la Société des Pathologies Exotiques, 89, 2, p. 115-119.

Girod R., Gaborit P., Carinci R., Issaly J., Fouque F., 2008. Anopheles darlingi bionomics and transmission of Plasmodium facilparum, Plasmodium vivax and Plasmodium malariae in Amérindian villages of the upper-Maroni Amazonian forest, French Guiana. Mem lnst Oswaldo Cruz, Rio de Janeiro, 103, 7, p. 702-710.

Hommel D., Ballandard F., Hulin A., 1997. Paludisme grave à Plasmodium falciparum. Aspects actuels en zone d'endémie guyanaise. Sem. Hop., Paris, 73, p. 197-2005.

Hong SK, Lee JA, 2006. Global environmental changes in terrestrial ecosystems. International issues and strategic solutions : introduction. Ecol. Res., 21, p. 783-787.

IEDOM, 2010. Les caractéristiques de l'habitat en Guyane. Note expresse, 65, 6 p.

InVS, 2006. Surveillance épidémiologique du paludisme en Guyane. 40 p.

INSEE, 2011, Projections de la population à l'horizon 2040. Plus d'un demi-million de Guyanais. Premiers Résultats, 71, $4 \mathrm{p}$.

INSEE, 2012, Populations légales en vigueur à compter du $1^{\mathrm{er}}$ janvier 2012. Recensement de la population. $7 \mathrm{p}$.

Juminer B., Robin Y, Pajot F.X., Eutrope R., 1981. Physionomie du paludisme en Guyane. Bull. Soc., Pathol. Exot., 74, p. 176-192.

Lepelletier L, Nadire-Galliot M., Poman J.P., Bellony S., Claustre J., Traore B.N, Mouchet J., 1989. Le paludisme en Guyane française. Situation de l'endémie. Bull. Soc. Pathol. Exot., 82, p. 385-392.

Leport C., Guégan J.-F. (dir.), 2011. Les maladies infectieuses émergentes : état de la situation et perspectives. Haut Conseil à la santé publique, la Documentation Française, coll. Evaluation, 208 p. Le Bras M., Malvy J.M. D., 2004. Le complexe pathogène tropical : regard nouveau sur un concept ancien. Médecine tropicale, 64, p. 613-618. 
Léone F., 2007. Caractérisation des vulnérabilités aux catastrophes "naturelles » contribution à une évaluation géographique multirisques (mouvements de terrain, séisme, tsunamis, éruptions volcaniques, cyclones). Mémoire d'HDR, vol.2, Université Paul Valéry, Montpellier 3, 245 p + annexes.

Léone F., Vinet F., 2007, La vulnérabilité, un concept fondamental au cœur des méthodes d'évaluation des risques naturels. Géorisques, 1, p. 9-25.

Lepelletier L., Gay F., Nadire-Galliot M., Poman J.p., Bellony S., Claustre J. 1989. Le paludisme en Guyane. Situation générale de l'endémie. Bull. Soc. Pathol. Exot., 82, p. 385-392.

Londres A., 2002. L'homme qui s'évada. Serpents à Plumes, coll. Motifs, réédition, 147 p.

Londres A., 2002, Au bagne. Serpents à Plumes, coll. Motifs, réédition, 247 p.

Meynard J.-B., Ardillon V., Venturin C., Ravachol F., Basurko C., Dussart Ph., Matheus S., Rabarison P., Gaborit P., Besançon L., Hervé A., Issaly J., Daudens E., Langevin S., Thalmensi G., Thauvin X., Dupuy B., Vantilcke V., Grenier C., Joubert M., Mansotte F., Djossou F., Morvan J., Quénel Ph., 2008. Première description d'une épidémie de dengue dans le commune de l'intérieur de la Guyane : Maripasoula, France, février 2006. Bul. épid. hebdo., 13, p. 93-96.

Ministère de l'Ecologie de l'Énergie, du Développement Durable et de la Mer, 2010. Orientation pour l'établissement public d'aménagement de Guyane (EPAG). Conseil Général de l'environnement et du Développement Durable. Rapport nº07254-01, mai 2010, 23 p.

Ministère de la Marine et des Colonies, 1942, Précis historique de l'expédition de Kourou (Guyane française) 1763-1765. Direction des Colonies, Paris, Imprimerie Royale, 87 p.

Morel V., Deboudt Ph., Hellequin A.-P., Herbert V., Meur-Férec C., 2006. Regard rétrospectif sur l'étude des risques en géographie à partir des publications universitaires (1980-2004).

L'Information Géographique, 70, p. 6-24.

Mouchet J., Nadire-Galliot M., Gay F., Poman J.P., Lepelletier L., Claustree J., Bellony S., 1989. Le paludisme en Guyane. Caractéristique des différents foyers et lutte antipaludique. Bull. Soc. Pathol. Exot, 82, p. 393-405.

Observatoire Régional de la santé de Guyane, 2010. Système de Santé: Guyane française, Brésil, Suriname. ORSG, 83 p.

Pajot F.-X., Le Font F., Molez J.-F., Dagallier N., 1977. Agressivité d'anophèles (Nyssorhynclus) darlingi Root, 1926 (Diptera, Culicidae) en Guyane française. Cahiers de l'ORSTOM, Série Ent. Méd et Parasito, XV, 1, p. 15-22.

Piantoni F., 2011. Migrants de Guyane. Arles, Actes sud, Musée des cultures guyanaises, 175 p.

Pierrat Ch., 2011. Risque palustre : appréhender la vulnérabilité des individus à l'échelle locale (Sud du Bénin). Vertigo, 11, 3, http://vertigo.revues.org/11549

Proust A., 1883. Le Choléra. Etiologie et Prophylaxie. Paris, Masson, 232 p.

Plowright R.T, Sokolow S.H., Gormam M.E., Daszack P., Foley J.E., 2008. Causal inference in desease ecology : investigating ecological drivers of disease emergence. Frontiers in Ecologie and the Environnement. 6, 8, p. 420-429.

Roll Back Malaria, 2011. $4^{e}$ journée mondiale de lutte contre le paludisme. Compte rendu des tables rondes «lutte contre le paludisme ». Ministère des Affaires Etrangères et Européennes, Roll Back Malaria, avril 2011, Paris, 28 p.

Reynes J.-M., 1996. La Dengue en Guyane française. Histoire et actualités. Bulletin de la Société des Pathologies Exotiques, 89, 2, p. 98-101. 
Salem, G., 1998. La santé dans la ville. Géographie d'un petit espace dense : Pikine (Sénégal). Karthala, $360 \mathrm{p}$.

Stéphani A., 2011. Epidémiologie du paludisme et environnement : étude de deux populations amérindiennes de l'est et de l'ouest guyanais. Thèse de doctorat en Sciences de la vie, Spécialité Santé publique. Université des Antilles et de la Guyane, 369 p.

\section{NOTES}

1. Les premières équipes chargées de la lutte antivectorielle en Guyane furent recrutées en 1947. Ces équipes furent constituées par arrêté du 26 novembre 1947 qui créa un cadre départemental d'agents d'hygiène assermentés. Les premiers traitements intra-domiciliaires avec le DDT furent pratiqués en 1949 (Cebret, Désiré, 1996).

2. Les situations épidémiologiques sont portées à connaissance via le Bulletin de Veille Sanitaire Antilles-Guyane (BVS) qui fait suite au Bulletin d'Alerte et de Surveillance Antilles Guyane (BASAG) publié par la CIRE des Antilles et de la Guyane

3. Le PSAGE (Programme de Surveillance d'Alerte et de Gestion des Epidémies) dengue de Guyane a été signé le 9 octobre 2010. Un PSAGE définit des stratégies de surveillance et de contrôle de la dengue graduées selon le risque épidémique, adapté à chaque charge département. Un double objectif: (1) contractualiser le rôle et les mission de chacun des partenaires impliqués dans le lutte contre la dengue (Préfecture, Conseil général, Municipalités, ARS, Hôpitaux, Médecins et laboratoires de ville, Forces Armées, Cire-InVS) s'engage à tenir ; (2) fournir les outils nécessaires pour la conduite des différentes actions du programme dans les domaines de la surveillance épidémiologique et entomologique, de la démoustication, de la communication et de la prise en charge des malades.

4. Le plateau des guyanes s'étend sur cinq pays d'est en ouest: le Venezuela, le Guyana, le Surinam, la Guyane française et le nord du Brésil jusqu'à l'Amazone.

5. Les virus de la dengue se classent en quatre types sérologiquement définis. On distingue donc les 4 sérotypes suivants: DEN-1, 2, 3 et 4 qui ne présentent pas d'immunité croisée entre eux au delà de quelques semaines.

6. CIRE : Cellule InterRégionale d'Epidémiologie.

7. Six groupes d'acteurs ont été identifiés: élus et collectivités, soignants, organismes de politique de santé, chercheurs, aménageurs, populations.

8. La mitigation recouvre les moyens et les mesures qui permettent d'atténuer les dommages sur les enjeux (environnementaux, sociaux, sanitaires,...) pour les rendre plus supportables par la société. La mitigation s'inscrit dans une démarche de gestion préventive des risques et est une composante de la stratégie du développement durable.

9. DFA : Départements Français d'Amérique

\section{RÉSUMÉS}

Vulnerability is a concept increasingly mobilized for understanding the emergence of situations of risk regardless of their nature (Beck, Glatron, 2009; Bogardi, 2004, D'Ercole, 1991; D'Ercole et al., 1994; Fabiani, Theys 1987; Fabiani, 2010, Leone 2007, Leone, Vinet, 2007, Pierrat, 2011). The 
use of vulnerability for the knowledge of conditions for the emergence risk of vector-borne diseases (VBD) opens a way for systemic and multidisciplinary analysis of risk that exceeds the knowledge of traditional social criteria such as age, gender, social class or level of income taken into account in epidemiological studies. If since the beginning of the nineteenth century, the anglo-aaxon work on the risks of VBD (Cohen, 2000; Daszack et al., 2000 and 2001; Hong, Lee, 2005; Midley, Thuiller, 2005, Plowright et al., 2008 ) take into account the vulnerability assessment, however the France lags behind the Haut Conseil de la santé publique wishes to catch up (Leport and Guégan, 2011). The study of territorial vulnerability of French Guiana to VBD opens new possibilities of action for the management of health risks.

\section{INDEX}

Keywords : vector-borne diseases (VBD), health hazard, territorial vulnerability, coastal zone, French Guiana

Mots-clés : maladie infectieuse à transmission vectorielle (MITV), risque, vulnérabilité territoriale, littoral, Guyane française

\section{AUTEUR}

\section{VALÉRIE MOREL}

Valérie Morel, valerie.morel@ird.fr / valerie.morel@univ-artois.fr, est Maître de conférences en Géographie, Université Lille Nord de France, Artois, EA2468 DyRT, et membre de l'UMR MIVEGEC IRD 224-CNRS UM1 et UM2 équipe DySMI, centre IRD de Cayenne 